\title{
AUTOMATIC EVALUATION OF THE INITIAL GEOPOSITIONING ACCURACY FOR LARGE AREA PLANETARY REMOTE SENSING IMAGES
}

\author{
Xun Geng ${ }^{1,2, *}$, Qing $\mathrm{Xu}^{2}$, Jiayao Wang ${ }^{1}$, Shuai Xing ${ }^{2}$ \\ ${ }^{1}$ Henan Industrial Technology Academy of Spatio-Temporal Big Data, Henan University, Kaifeng 475004, China. - \\ gengxun_rs@163.com \\ ${ }^{2}$ Dept. of Photogrammetry, Zhengzhou Institute of Surveying and Mapping, Zhengzhou 450052, China
}

Commission III, ICWG III/II

KEY WORDS: Planetary photogrammetry, Mars mapping, Mars Express, Image matching, Orthorectification

\begin{abstract}
:
The photogrammetric processing of large area planetary remote sensing images is still a very challenging work. In addition to the lack of ground control data and poor tie points extraction, the insufficient knowledge of the initial geopositioning accuracy of the planetary images also increases the difficulty of processing. This paper presents an automatic evaluation method of the initial geopositioning accuracy for large area planetary remote sensing images. The accuracy evaluation method was conducted through image matching on approximate orthophotos derived using coarse resolution digital elevation model (DEM). To improve the orthophotos generation efficiency of linear pushbroom images, a fast ground-to-image transformation algorithm and multi-threaded parallel computing are adopted. The classical normalized cross correlation (NCC) and pyramid matching schemes are used to perform image matching between overlapping orthophotos. Because the conjugate points on orthophotos contain geographic coordinates, we can derive the statistics information (e.g., maximum errors, mean errors and standard deviation) about the geopositioning accuracy of the planetary images. Although it's actually an evaluation result of relative accuracy, the quantitative geopositioning accuracy information of stereopairs can be used to (1) specify the search window size and the starting position of conjugate points for tie points extraction; (2) set the weight value of bundle adjustment; and (3) identify images with abnormal geopositioning accuracy. Tens of Mars Express (MEX) High Resolution Stereo Camera (HRSC) images were used to conduct the test. The experimental results demonstrate that the proposed method shows high computational efficiency and automation degree. The automatic evaluation of the initial geopositioning accuracy of the planetary images is helpful to produce large area planetary mapping products.
\end{abstract}

\section{INTRODUCTION}

Large area planetary mapping products such as seamless mosaicked Digital Orthophoto Map (DOM) and Digital Elevation Model (DEM) can be used to select landing sites and assess landing safety, which is also the basic geographic information data for planetary science (Kirk et al., 2008; Wu et al., 2014; Di et al., 2019). However, existing planetary mapping products can not meet the needs of deep space exploration engineering and planetary scientific research. For example, the spatial resolution of Mars global mapping products is still at the level of $100 \mathrm{~m}$ (Putri, et al., 2019). Although the recent space exploration missions have returned massive planetary remote sensing images, most of them have not been strictly processed geometrically. More specifically, the released Mars Express (MEX) High Resolution Stereo Camera (HRSC) Level 4 products were derived by single strip bundle adjustment, such that there are several or dozens of pixels offsets between adjacent strips (Gwinner et al., 2016). In theory, the fusion processing of planetary images acquired by different space agencies can derive planetary mapping products with higher resolution and higher accuracy. Take Mars mapping for example, the fusion processing of planetary images returned by MEX HRSC, MRO CTX as well as the upcoming China's first Mars exploration mission (i.e., Tianwen-1) can generate Mars global DOM with a spatial resolution of $10 \mathrm{~m} /$ pixel and Mars global DEM with a spatial resolution of better than $50 \mathrm{~m} /$ pixel. However, fusion processing of massive planetary images shows great technical difficulty. Therefore, it is very meaningful to develop the photogrammetric processing techniques to solve the key problems in geometric processing of large area planetary images (Kirk et al., 2017; Geng et al., 2019).

It is noted that the released SPICE kernels show different geopositioning accuracy levels (e.g., prediction and reconstruction levels). Moreover, the auxiliary data of planetary images with long time span always show inconsistency. Thus, even if the stereo images have SPICE kernels with reconstruction level accuracy, there may be large inconsistencies in the overlapping area. Due to the lack of ground control data, the bundle adjustment results of planetary images greatly depend on the initial geopositioning accuracy. But traditional photogrammetric processing techniques usually don't make a quantitative analysis of the initial geopositioning accuracy of the planetary images, such that the bundle adjustment process needs to try different parameters setting based entirely on past experiences. Consequently, it will require more iterations to derive a satisfactory bundle adjustment result. Moreover, very few abnormal images will destroy the overall adjustment results and need to be identified in advance. Unfortunately, it is difficult to identify the images with abnormal geopositioning accuracy by using metadata information alone. Although single strip bundle adjustment or manual measurements of some conjugate points in the overlapping area can increase our knowledge about the initial

\footnotetext{
* Corresponding author
} 
geopositioning accuracy of the planetary images, it is inefficient and involves a lot of manual operations.

This paper presents a method for automatically evaluating the initial geopositioning accuracy of planetary images. The image matching methodology based on approximate orthophotos is used to analyse the geographic coordinates offsets of stereo images.

\section{INITIAL GEOPOSITIONING ACCURACY EVALUATION}

To evaluate the initial geopositioning accuracy of the planetary images, we first generate the approximate orthophotos of raw planetary images, and then conduct image matching of each stereopair using the derived orthophotos. The initial geopositioning accuracy of planetary images can be derived through computing the statistics information of the matching results. The schematic diagram of the proposed method is shown in Figure 1.

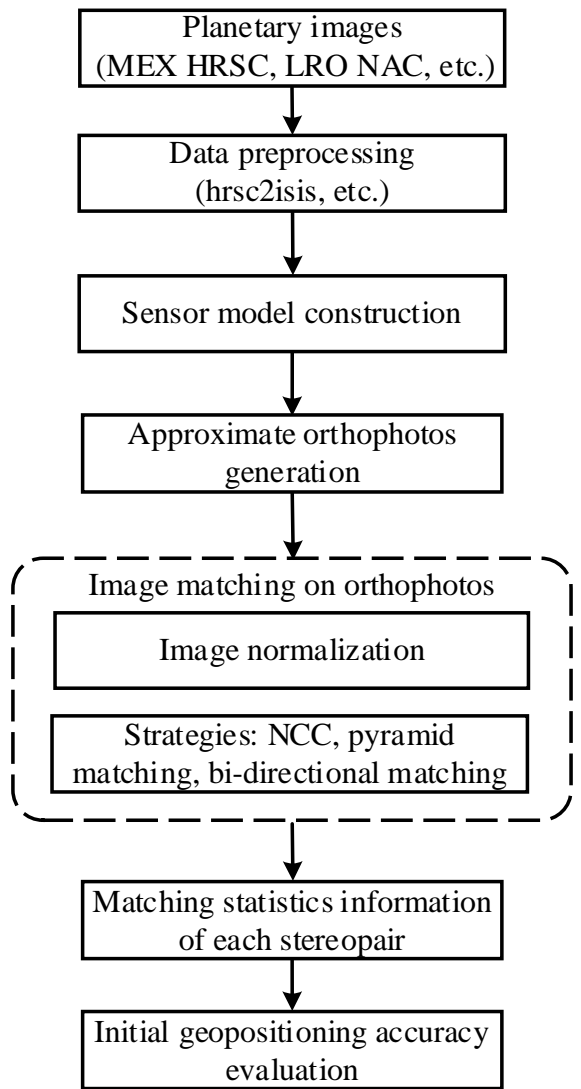

Figure 1. The flow chart of evaluating the initial geopositioning accuracy of planetary images.

The detailed processing procedures are as follows:

1. Import the raw planetary images into the Integrated Software for Imagers and Spectrometers (ISIS) developed by the United States Geological Survey (USGS) Astrogeology team (Edmundson et al., 2012) and then conduct data preprocessing (e.g., spiceinit).

2. Extract the interior and exterior orientation parameters of each image and then construct the sensor model.

3. Generate approximate orthophotos using low resolution DEM such as Mars Orbiter Laser Altimeter (MOLA) DEM.
4. Conduct image matching based on approximate orthophotos. Firstly, image normalization is conducted to derive stereo images with the same ground sample distance (GSD). Then, the classical normalized cross correlation (NCC) matching method is used to acquire evenly distributed conjugate points. To enhance the reliability and robustness of the matching results, pyramid matching and bi-directional matching strategies are used.

5. Compute the statistics information of the image matching results for each stereopair.

6. The derived statistics information can be used to evaluate the initial geopositioning accuracy of the planetary images.

In the procedure of sensor mode construction, we adopt a generic pushbroom sensor model developed for planetary images (Geng et al., 2020). To improve the computational efficiency of orthophotos generation, a fast ground-to-image transformation algorithm of linear pushbroom images is used (Geng et al., 2019). With the image matching results of approximate orthophotos, the geographic coordinates difference of the conjugate points can be computed. Then, the statistics information of matching for each stereopair can be derived, including maximum errors, mean errors and standard deviation.

\section{RESULTS AND DISCUSSION}

\subsection{Test Data}

We selected 30 strips of MEX HRSC images in one of the potential landing areas of China's first Mars exploration mission to conduct experiments (see Figure 2). The HRSC stereo images acquired by the same strip (e.g., the forward-looking s12 and backward-looking s22) always show consistent geopositioning accuracy. But we are mainly concerned about the geopositioning accuracy of stereo images acquired by different strips. Thus, only the downward-looking panchromatic channel (i.e., nd2) of the HRSC images was used in the test. The GSD of the test images varies between $13.4 \mathrm{~m}$ and $22.5 \mathrm{~m}$. The image acquisition date was from Dec. 2007 to Jan. 2018. The basic information of the test data is shown in Table 1.

The USGS ISIS is used to conduct pre-processing. The software modules for orthophotos generation and image matching were developed by us using Qt 5.2.1 in the Ubuntu 14.04 operating system. The hardware configurations were an Intel Core i7$7500 \mathrm{U}$ with a $2.70 \mathrm{GHz} \mathrm{CPU}$ and $8 \mathrm{~GB}$ RAM. The radius of the reference sphere is $3396.19 \mathrm{~km}$ for Martian images. In addition, we also developed a script program to automate all calculation steps, including preprocessing (i.e., importing raw images and sensor model construction), orthophotos generation and image matching. Thus, we only need to adjust some necessary parameters such as NCC threshold value and image pyramid levels in the practical processing procedures.

\begin{tabular}{|c|c|}
\hline Number of strips & 30 \\
Ground sample distance & $13.4 \sim 22.5 \mathrm{~m}$ \\
Image acquisition date & $2007.12 \sim 2018.01$ \\
data volume of Level2 image products & $15.1 \mathrm{~GB}$ \\
SPICE kernels quality & reconstructed \\
Orthophotos generation time & 59 minutes \\
Image matching time & 65 minutes \\
\hline
\end{tabular}

Table 1. Basic information of the test data. 


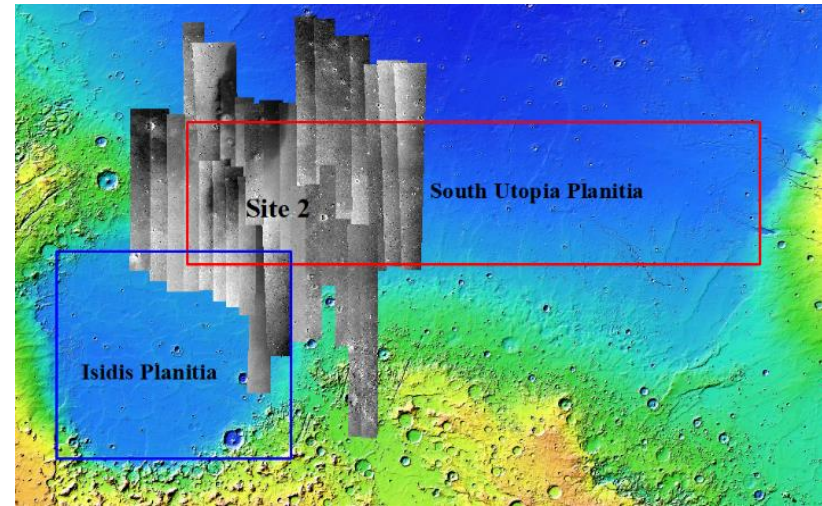

Figure 2. Test data in one of the potential landing areas of China's first Mars exploration mission. The red and blue rectangles indicate the potential landing areas in the South Utopia Planitia and Isidis Planitia respectively.

\subsection{Initial Geopositioning Accuracy Evaluation Results}

The number of matched conjugate points for each stereopair varies from several to hundreds as shown in Figure 3(a), which is mainly affected by the range of the overlapping areas. In addition, in order to increase the coverage, some images with incidence angels greater than 70 degrees were used in the test. Therefore, the planetary images with poor illumination conditions resulted in fewer conjugate points. The maximum errors in the sample and line directions were less than $200 \mathrm{~m}$ for most stereopairs, as can be seen in Figure 3(b). Figure 3(c) shows that the absolute mean errors in the sample and line directions were less than $150 \mathrm{~m}$. This indicates that the average geometric offsets between stereo orthophotos are only a few pixels. The standard deviation of the stereopairs also shows interesting results. As shown in Figure 3(d), most of the standard deviation values were less than $50 \mathrm{~m}$. Figure 3(e) and (f) illustrate an example of the image matching results for a stereopair. As can be seen, there are no obvious mismatches.

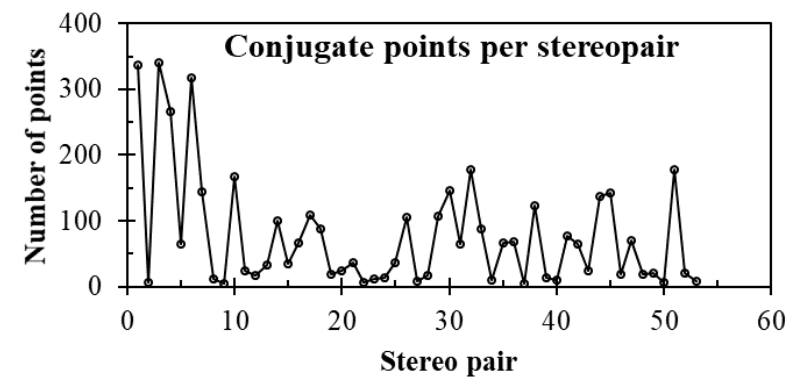

(a)

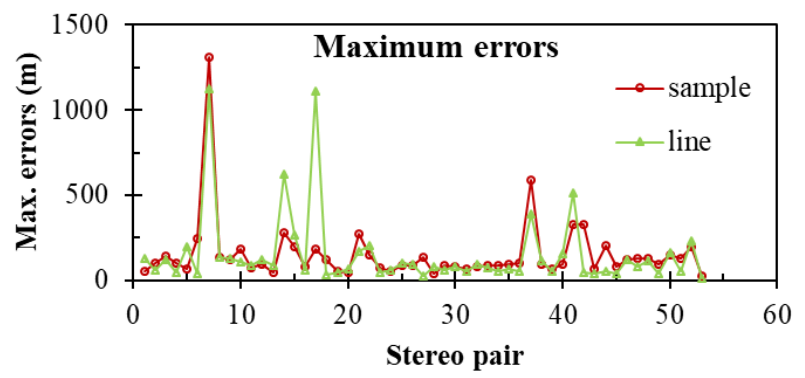

(b)

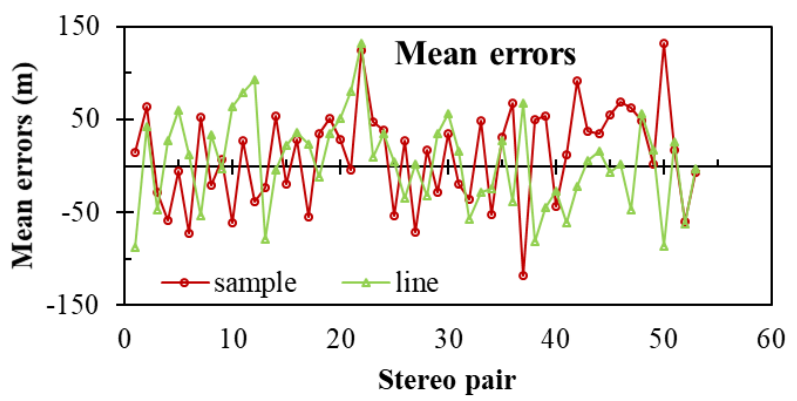

(c)

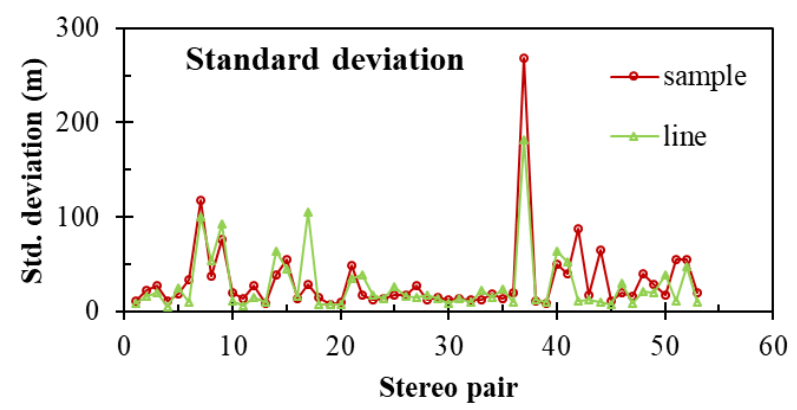

(d)

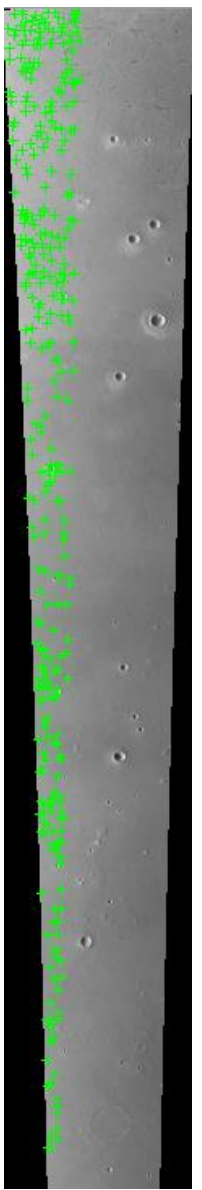

(e)

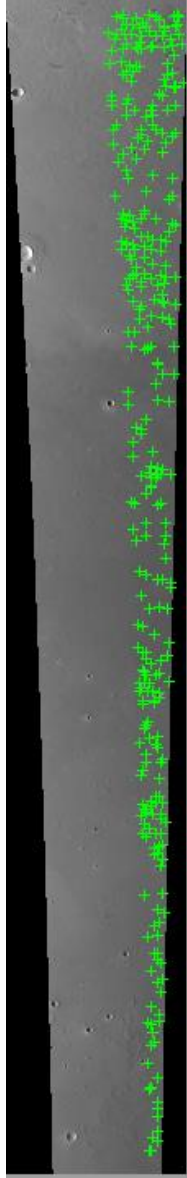

(f)
Figure 3. Evaluation results of the initial geopositioning accuracy of the test data. (a) the number of conjugate points per stereopair; (b) maximum errors between stereo images; (c) mean errors between stereo images; (d) standard deviation between stereo images; (e) conjugate points drawn on $\mathrm{h} 5097 \mathrm{nd} 2$ orthophoto; and (f) conjugate points drawn on h5115 nd2 orthophoto. 


\subsection{Discussion}

The statistics information of the image matching indicates that the image distortions are greatly removed by orthorectification, such that the remaining errors in the conjugate points are mainly systematic errors. This is very useful to specify the search window size and the starting position of conjugate points in the tie points extraction process. Furthermore, the weight values of unknowns in the bundle adjustment process can be specified based on the statistics information as well, which can provide a more reasonable adjustment range compared with using only past experience values.

Generally, the initial geopositioning accuracy of the test images is relatively high. It should be noted that such high accuracy is mainly due to the fact that the reconstructed SPICE kernels are used. If only the predicted SPICE kennels are available, the situation will be different. In addition, the very few stereopairs with poor geopositioning accuracy need further analysis, indicating that these images should be processed separately. Obviously, removing problematic images from the image lists is better than just putting all images into one bundle adjustment solution in the practical large area photogrammetric projects. The experimental results demonstrate that the quantitative evaluation of the initial geopositioning accuracy for dozens of planetary images can be completed within several hours, as shown in Table 1. Therefore, the proposed method has high computational efficiency and automation degree.

\section{CONCLUSION}

In order to realize the automatic processing of massive planetary images, it is necessary to clearly know the initial geopositioning accuracy of these images before adjustment. Through image matching on approximate orthophotos, the initial geopositioning accuracy of planetary images can be quantified in an automatic manner. Based on the experimental results, we recommend that the proposed accuracy evaluation method should be used prior to conduct the bundle adjustment process, and the derived statistics information of stereopairs can be used as a posterior experience to facilitate the bundle adjustment processing. It will help with the regional or global planetary mapping work involving hundreds or even thousands of images. Though the accuracy evaluation method was tested using HRSC images, it is obvious that it also applies to other planetary bodies with solid surface, which do not contain man-made or moving objects. Furthermore, if the refined EO parameters are used to derive orthophotos, the proposed method can also be used as an accuracy evaluation index to assess the final bundle adjustment results.

\section{ACKNOWLEDGEMENTS}

This study was supported by the National Natural Science Foundation of China with project number 41401533 . We thank the MEX HRSC team for providing the planetary images to the public. We also thank the authors of the open source planetary photogrammetric software packages-ISIS.

\section{REFERENCES}

Di, K., Jia, M., Xin, X., Wang, J., Liu, B., Li, J., Xie, J., Liu, Z., Peng, M., Yue, Z., et al., 2019. High-resolution large-area digital orthophoto map generation using LROC NAC images. Photogramm. Eng. Remote Sens., 85, 481-491.

Edmundson, K.L., Cook, D.A., Thomas, O.H., Archinal, B.A., Kirk, R.L., 2012. Jigsaw: the ISIS3 bundle adjustment for extraterrestrial photogrammetry. ISPRS Ann. Photogramm. Remote Sens. Spatial Inf. Sci., I-4, 203-208. doi.org/10.5194/isprsannals-I-4-203-2012

Geng, X., Xu, Q., Xing, S., Lan, C., 2019. A robust ground-toimage transformation algorithm and its applications in the geometric processing of linear pushbroom images. Earth Space Sci., 6, 1805-1830.

Geng, X., Xu, Q., Xing, S., Lan, C., 2020. A generic pushbroom sensor model for planetary photogrammetry. Earth Space Sci., 7 , in press.

Gwinner, K., Jaumann, R., Hauber, E., Hoffmann, H., Heipke, C., Oberst, J., Neukum, G., Ansan, V., Bostelmann, J., Dumke, A., et al., 2016. The High Resolution Stereo Camera (HRSC) of Mars Express and its approach to science analysis and mapping for Mars and its satellites. Planet. Space Sci., 126, 93-138.

Kirk, R.L., Kraus, E.H., Rosiek, R.M., Anderson, J.A., Archinal, B.A., Becker, K.J., Cook, D.A., Galuszka, D.M., Geissler, P.E., Hare, T.M, et al., 2008. Ultrahigh resolution topographic mapping of Mars with MRO HiRISE stereo images: meter-scale slopes of candidate Phoenix landing sites. J. Geophys. Res. Planet., 113, 5578-5579.

Kirk, R.L., Howington-Kraus, E., Edmundson, K., Redding, B., Galuszka, D., Hare, T., Gwinner, K., 2017. Community tools for cartographic and photogrammetric processing of Mars Express HRSC images. Int. Arch. Photogramm. Remote Sens. Spatial Inf. Sci., XLII-W1, 69-76. doi.org/10.5194/isprs-archives-XLII-3W1-69-2017.

Putri A.R.D., Sidiropoulos P., Muller J.P., Walter S.H.G., et al., 2019. A new south polar digital terrain model of Mars from the High-Resolution Stereo Camera (HRSC) onboard the ESA Mars Express. Planetary Space Sci., 174, 43-55.

Wu, B., Hu, H., and Guo, J., 2014. Integration of Chang'E-2 imagery and LRO laser altimeter data with a combined block adjustment for precision lunar topographic modeling. Earth Planet. Sci. Lett., 391, 1-15. 